\title{
Ischemic Stroke in Patients with Cancer: A Retrospective Cross-Sectional Study
}

\author{
Anca Motataianu ${ }^{1}$, Smaranda Maier ${ }^{1}$, Sebastian Andone ${ }^{2}$, Laura Barcutean ${ }^{1}$, Georgiana \\ Serban², Zoltan Bajko ${ }^{*}$, Adrian Balasa ${ }^{1}$ \\ ${ }^{1}$ George Emil Palade University of Medicine, Pharmacy, Science, and Technology of Targu Mures, Romania \\ 2 Emergency County Hospital Targu Mures, Romania
}

\begin{abstract}
Introduction: An increasing trend of cancer associated stroke has been noticed in the past decade. Objectives: To evaluate the risk factors and the incidence of neoplasia in stroke patients. Material and Method: A retrospective, observational study was undertaken on 249 patients with stroke and active cancer (SAC) and 1563 patients with stroke without cancer (SWC). The general cardiovascular risk factors, the site of cancer, and the general clinical data were registered and evaluated. According to the "Oxfordshire Community Stroke Project" (OCSP) classification, all patients were classified into the clinical subtypes of stroke. The aetiology of stroke was considered as large-artery atherosclerosis, small vessel disease, cardio-embolic, cryptogenic or other determined cause. Results: The severity of neurological deficits at admission were significantly higher in the SAC group $(p<0.01)$. The haemoglobin level was significantly lower, and platelet level and erythrocyte sedimentation rate were significantly higher in the SAC group. Glycaemia, cholesterol and triglycerides levels were significantly higher in the SWC group. The personal history of hypertension was more frequent in the SWC group. In the SAC group, $28.9 \%$ had a cryptogenic aetiology, compared to 9.1\% in SWC group. Cardio-embolic strokes were more frequent in the SAC group (24\%) than the SWC group (19.6\%). In the SAC group, $15,6 \%$ were diagnosed with cancer during the stroke hospitalization, and $78 \%$ of the SAC patients were without metastasis. Conclusions: The most frequent aetiologies of stroke in cancer patients were cryptogenic stroke, followed by large-artery atherosclerosis. SAC patients had more severe neurological deficits and worse clinical outcomes than SWC patients. Stroke in cancer patients appears to be more frequently cryptogenic, probably due to cancer associated thrombosis. The association between stroke and cancer is important, especially in stroke of cryptogenic mechanism, even in the presence of traditional cardiovascular risk factors.
\end{abstract}

Keywords: cancer, stroke, cancer associated thrombosis, cryptogenic stroke, cardiovascular risk factors

Received: 21 September 2020 / Accepted: 5 January 2021

\section{INTRODUCTION}

Armand Trousseau first described thrombosis as a cancer complication more than one hundred and fifty years ago; however, the approach to the treatment of cancer patient with thrombotic events still presents a clinical challenge [1]. In the past three decades, anatomic-pathological and clinical studies revealed an increasing trend in cancer associated arterial thrombosis, including ischemic stroke $[2,3]$. Cancer-associated thrombosis (CAT) has attracted significant interest in recent years, especially concerning the involved mechanisms and the potential biomarkers, to define optimal therapeutic strategies.

After cerebral metastasis, ischemic stroke is the second most common complication involving the central nervous system in cancerous patients. Almost $10 \%$ of the patients with ischemic stroke have a personal history of neoplasia, and this clinical association seems to be more frequent than previously described $[4,5,6]$. Due to the significant progress made in cancer diagnosis and treatment in the last years, most of these patients will survive for over five years following a positive diagnosis [7].

Therefore, it is crucial to prevent thrombotic complications to maintain the quality of life and enhance the survivability of these patients.

Neoplasia has become increasingly recognized as a risk factor for stroke in the past ten years.

The risk of ischemic stroke is higher in cancer patients, being conditioned by the level of aggressiveness 
and the clinical stage of the neoplasia [8]. Occasionally, stroke can be the first clinical sign of occult neoplasia [9].

The diagnosis of stroke in cancer patients aggravates the prognostic and increases the caregiver burden [10].

The aetiopathogenesis of stroke in cancer patients is complex, with a broad spectrum of variability, and differs in cancer-free patients. In patients with stroke and active cancer, neoplasia associated with a hypercoagulation state and the administration of oncological treatment, co-exist with traditional cardiovascular risk factors $[3,8,11,12]$.

Tumour cells produce various cytokines which directly or indirectly activate the coagulation cascade and inhibit the anticoagulating activity, thus producing stroke in cancer patients [13].

This study aimed to evaluate two groups of newly diagnosed stroke patients, one group being cancer free and the other having associated cancer, from the perspective of neurological deficits, clinical outcome and paraclinical biomarkers.

While the association between neoplasia and traditional stroke risk factors is not uncommon, it is hypothesized that cancer diagnosis is directly linked to stroke risk in specific cases. Thus, the null hypotheses are:

- There is no difference regarding neurological deficits between stroke patients with associated cancer and stroke patients without cancer.

- There is no difference regarding clinical outcome at discharge between stroke patients with associated cancer and the stroke patients without cancer.

\section{MATERIAL AND METHODS}

Using the departmental stroke database, 1812 patients with ischemic stroke, admitted to the $1^{\text {st }}$ Department of Neurology-Stroke Center, Emergency Clinical County Hospital of TarguMures, Romania, from 01.10.2009 to 01.10.2019 were identified.

Two hundred forty-nine patients with stroke and active cancer diagnosis, or those who were diagnosed with cancer during stroke hospitalization and 1563 patients with stroke and without active cancer.

From all the patients with ischemic stroke, we selected 249 patients with stroke and active cancer diagnosis or those diagnosed with cancer during stroke hospitali- zation and 1563 patients with stroke and without active cancer.

All the data was gathered using the official medical records, backed-up by histological and oncological evidence.

The following clinical and demographical characteristics were recorded:

- age

- gender

- height

- weight

- blood pressure on admission

- heart rate

The following cardiovascular risk factors were registered:

- hypertension

- dyslipidaemia

- diabetes mellitus

- coronary artery disease

- smoking and alcohol consumption before the stroke

- prior myocardial infarction

- peripheral artery disease

- paroxysmal or chronic atrial fibrillation (AF).

On the day of admission, a neurological examination was performed according to the hospital's standardized care protocol for stroke patients and was documented using the National Institutes of Health Stroke Scale (NIHSS) and the level of consciousness. The modified Rankin Scale (mRs) was noted at discharge.

The diagnostic workup outlined the paraclinical investigations necessary to establish the stroke diagnosis, the assessment of risk factors and evaluation of said risk factors, and yes, it is in addition to the neurological examination.

On admission, a cerebral computed tomography (CT) scan or magnetic resonance imaging (MRI) and an electrocardiography (ECG) followed by seventy-two hours of vital signs and ECG monitoring.

On admission, all patients underwent a cerebral computed tomography (CT) scan or magnetic resonance imaging (MRI), electrocardiography (ECG), followed by seventy-two hours of vital signs and ECG monitoring. Basic emergency laboratory tests including haematology, erythrocyte sedimentation rate (ESR), routine biochemistry, cholesterol and triglyc- 
erides level and standard coagulation tests including prothrombin time, international normalized ratio and activated partial thromboplastin time were taken and recorded.

During hospitalization, all patients underwent extra- and transcranial Doppler ultrasonography of carotid and vertebral arteries and transthoracic echocardiography.

According to the "Oxfordshire Community Stroke Project” (OCSP) classification, all patients were classified into the clinical subtypes of stroke.

Patients were classified as having a total anterior circulation infarct (TACI), partial anterior circulation infarct (PACI), posterior circulation infarct (POCI) or a lacunar infarct (LACI).

According to the "Trial of Org 10172 in Acute Stroke Treatment" (TOAST) classification, the stroke aetiologies were determined by the attending neurologist. This classification includes the aetiology of stroke as large-artery atherosclerosis, small vessel disease, cardio-embolic, other determined cause or undetermined (cryptogenic) cause of stroke $[14,15]$.

Neoplastic history was assessed as being active or inactive. Active cancer was defined as: (1) confirmed and active-treated malignancy (2) confirmed but untreated malignancy in the six months before the stroke; (3) new cancer diagnosis during stroke hospitalization (4) metastasis of known cancer (5) recurrent cancer.

SAC patients with inactive cancer, i.e.personal history of neoplasia for more than ten years, healed neoplasia, the uncertainty of the diagnosis, and intracranial malignancies, were excluded.

The prior diagnosis of cancer was confirmed by referencing the patient's history, as reported in official oncology documents. The new cancer diagnosis, i.e. recent diagnosis during hospitalization, was established using computer tomography (CT), magnetic resonance imaging (MRI), clinical, serological and histological assessments.

The statistical analysis was performed using Graph Pad Prism 5.0. The data values were expressed as mean (SD) when assessing Gaussian data.

The level of significance was set at $\alpha=0.05$ for all tests.

Patients were divided into two groups: the SAC - Stroke patients with Active Cancer, and the SWC Stroke patients Without cancer.
Student's t-test and Chi-square test were used for the univariate analyses when appropriate.

For multivariate analyses, binary logistic regression analysis was used.

\section{RESULTS}

The study population consisted of 249 patients with stroke and active cancer (SAC) and 1563 patients with stroke but without active cancer (SWC). The mean age (SD) in the SAC group was 71.7 (9.9) years, and for SWC, it was 70.2 (12.4 years). The demographical, clinical and para-clinical data are presented in Table 1.

There were no significant differences between the two groups in age distribution, but there was a statistically significant difference in gender distribution (Fischer's exact test; $\mathrm{p}<0.01$ ).

The length of hospitalizations did not significantly differ between the two groups (Student's t-test; $\mathrm{p}>0.05$ ).

The systolic blood pressure and diastolic blood pressure were significantly higher in the SAC group upon admission. (Student's t-test; $\mathrm{p}<0.01$ ).

The haemoglobin level was significantly lower in the SAC group (Student's t-test; $\mathrm{p}<0.01$ ).

The thrombocyte number and ESR were significantly higher in the SAC group (Student's t-test; $\mathrm{p}=0.03$, $\mathrm{p}<0.01)$. The glycaemia, cholesterol and triglycerides levels were significantly higher in the SWC group (Student's t-test; $\mathrm{p}<0.01$ in each case).

The severity of stroke neurological deficits at admission, assessed by NIHSS and clinical outcome at discharge, assessed by modified Rankin scale were significantly higher in the SAC group (Student's t-test; $\mathrm{p}<0.01)$.

Regarding the cardiovascular risk factors analysis, there was no significant difference in smoking habits and alcohol consumption. There was a statistically significant difference between diabetes and AF between the two groups (Fisher's exact test; $\mathrm{p}<0.01$ ).

The presence of hypertension in the personal medical history was more frequent in the SWC group (Fisher's exact test; $\mathrm{p}<0.01)$.

Of 249 the SAC patients, 72 (28.9\%) had an unknown or cryptogenic aetiology, compared to $143(9.1 \%)$ of the 1563 SWC patients. Large-artery atherosclerosis was found in $28.1 \%$ of the SAC patients and $42.8 \%$ of the 
Table 1. Demographical, clinical and laboratory characteristics of stroke patients with cancer and without cancer

\begin{tabular}{|c|c|c|c|}
\hline & SAC (N=249) & SWC ( $N=1563)$ & P-value \\
\hline Age & $71.70(9.98)$ & $70.24(12.473)$ & 0.08 \\
\hline Gender (M: F) & 155:94 & $807: 756$ & $<0.01$ \\
\hline Hospitalization days & $10.07(5.219)$ & $10.72(7.497)$ & 0.19 \\
\hline NIHSS on admission & $6.41(5.274)$ & $4.93(5.398)$ & $<0.01$ \\
\hline GCS on admission & $14.73(1.143)$ & $14.64(1.593)$ & 0.48 \\
\hline mRS at discharge & $2.66(1.492)$ & $2.21(2.299)$ & $<0.01$ \\
\hline SBP on admission & $159.86(29.756)$ & $148.63(32.376)$ & $<0.01$ \\
\hline DBP on admission & $89.35(17.805)$ & $85.44(16.395)$ & $<0.01$ \\
\hline Cholesterol & $173.21(46.327)$ & $184.22(47.852)$ & $<0.01$ \\
\hline Triglycerides & $127.87(66.191)$ & $145.96(108.627)$ & 0.03 \\
\hline Thrombocytes & 255431(116888) & 239133(110479) & 0.03 \\
\hline Haemoglobin & $12.78(2.309)$ & 13.7851(1.87791) & $<0.01$ \\
\hline Glycemia & $122.48(41.718)$ & 129.63(49.251) & 0.03 \\
\hline ESR & $30.00(28.901)$ & $18.70(18.255)$ & $<0.01$ \\
\hline
\end{tabular}

Abbreviations: SAC-stroke and cancer; SWC -stroke without cancer; ESR- erythrocyte sedimentation rate; SBP-systolic blood pressure; DBP-diastolic blood pressure; NIHSS - the National Institutes of Health Stroke Scale; mRS-modified Rankin scale; GCS-Glasgow Coma Scale

SWC patients. Cardioembolic strokes were significantly more frequent in the SAC group (24\%) versus SWC group (19.6\%) (Fisher's exact test; $\mathrm{p}=0.01$ ).

Total anterior circulation infarct was present in 40 (16\%) SAC patients and 300 (19.1\%) SWC patients (Table 2).

From all patients in the SAC group, 15,6\% were diagnosed with cancer during hospitalization for stroke, and $78 \%$ of the SAC group were without metastasis.
In the 249 SAC patients, the most frequent localization of the primary cancer was the lungs, 48 (19.28\%), followed by prostate and colon in $23(9.24 \%)$ cases, urinary bladder in $22(8.84 \%)$ cases, breast in $21(8,43 \%)$ cases, gynaecological and haematological in 20 (8.03\%) cases. Ear-nose-throat-related neoplasia was found in $18(7.23 \%)$ cases, gastric cancer in $13(5.22 \%)$ cases, and pancreatic cancer in $11(4.42 \%)$ cases. Skin, renal, thyroid and other cancers were recorded in less than $5 \%$ of the cases. The mean (SD) age of the patients diag-

Table 2. Characteristics of the study patients according to the cardiovascular risk factors, stroke aetiology based on TOAST classification and cerebral territory involved based on the OCSP criteria.

\begin{tabular}{lccc} 
& SAC (N=249) & SWC (N=1563) & P-value \\
Risk factors & & & \\
Hypertension history (Y: N) & $196: 53$ & $1464: 99$ & $<0.01$ \\
Smoking history (Y: N) & $48: 177$ & $376: 1187$ & 0.36 \\
Alcohol consumption history (Y: N) & $46: 176$ & $394: 1169$ & 0.14 \\
Diabetes mellitus (Y:N) & $48: 201$ & $242: 1321$ & $<0.01$ \\
AF (Y: N) & $60: 189$ & $257: 1306$ & $<0.01$ \\
Seizures (Y: N) & $11: 238$ & $38: 1525$ & 0.07 \\
TOAST classification & & & \\
Large artery atherosclerosis & 70 & 669 & $<0.01$ \\
Small vessel disease & 41 & 224 & 0.92 \\
Cardioembolic & 60 & 306 & 0.01 \\
Other known cause & 6 & 48 & 0.56 \\
Cryptogenic cause & 72 & 143 & $<0.01$ \\
OCSP classification & & & \\
TACl & 40 & 300 & 0.04 \\
PACl & 135 & 691 & 0.23 \\
POCl & 58 & 298 & 0.55 \\
LACl & 6 & 33 & 1.00 \\
\hline
\end{tabular}


nosed with lung cancer was 66.94 (10.69). Prevalence of different types and organ location of the malignancy are given in Table 3.

The adjusted multivariate logistic regression model analysis regarding independent risk factors showed that stroke patients with high cholesterol level, diabetes without hypertension and smoking history were more likely to be associated with the presence of cancer (Table 4).

\section{DISCUSSION}

An increasing number of studies report the risk of ischemic stroke in cancer patients. Recently, Jang et al. (2019) demonstrated that in a long-term follow-study, the risk is higher in the first three years following cancer diagnosis and that the same risk is maintained for the next seven years [16]. Navi et al. (2015) reported that cancer diagnosis is associated with a short-time increased risk for stroke, especially in colorectal, pancreatic and lung cancer [4]. The recent advances in can-

Table 4. Multivariate logistic regression analysis

\begin{tabular}{lccc} 
& p-value & \multicolumn{2}{c}{ 95\%C.I.for EXP(B) } \\
Lower & Upper \\
Cholesterol & 0.000 & 1.002 & 1.008 \\
Triglycerides & 0.819 & 0.997 & 1.002 \\
Thrombocytes & 0.184 & 1.000 & 1.000 \\
Diabetes mellitus & 0.001 & 1.357 & 3.563 \\
Hypertension history & 0.000 & 0.026 & 0.057 \\
Smoking history & 0.006 & 0.216 & 0.777 \\
Alcohol history & 0.193 & 0.395 & 1.207 \\
AF & 0.149 & 0.873 & 2.444 \\
\hline
\end{tabular}

Table 4. Primary cancer location in stroke patients

\begin{tabular}{lcc} 
Affected organ & Nr. & \% \\
Colon & 23 & $9.24 \%$ \\
Haematological & 20 & $8.03 \%$ \\
Breast & 21 & $8.43 \%$ \\
Urinary bladder & 22 & $8.84 \%$ \\
Prostate & 23 & $9.24 \%$ \\
Lung & 48 & $19.28 \%$ \\
Pancreas & 11 & $4.42 \%$ \\
Skin & 7 & $2.81 \%$ \\
Gynaecological-related & 20 & $8.03 \%$ \\
Gastric & 13 & $5.22 \%$ \\
Ear-nose-throat-related & 18 & $7.23 \%$ \\
Renal & 9 & $3.61 \%$ \\
Thyroid & 6 & $2.41 \%$ \\
Other organs & 8 & $3.21 \%$ \\
\hline
\end{tabular}

cer diagnosis and management have led to an increased survival rate, to more than five years after the onset. Thus, careful prevention of cancer-related complications is needed to maintain an optimal functional status and a good quality of life for these patients $[5,7]$.

In our study, it was found that SAC was significantly higher in male patients. This data is in accordance with the OASIS-CANCER study [17]. Khorana et al. (2006) found that male patients with cancer are more prone to developing arterial thrombotic events, whereas female patients with cancer are more likely to have venous thromboembolic events [18]. Selvik et al. (2015) reported that patients with neoplasia were older than the control group patients, but the patients diagnosed with lung cancer were significantly younger than the control group [19]. The reported mean age in the present study, diagnosed with lung cancer (SWC ) was 66.94 (10.69), which compared favourably with the 68.4 (11.2) age in a similar group of patients reported by Selvik.

The risk of ischemic stroke is higher in patients with neoplasia due to multiple causes. The contributing factors might be: (1) a hypercoagulation state produced by the tissue factor, tumoral procoagulant and proinflammation cytokines secreted by the tumour cells; (2) chemotherapy, especially the platinum-based type and with angiogenesis inhibitors which determine the endothelial activation and the extravasation of microparticles from the tumoral cells, that subsequently stimulate thrombin genesis; (3) vasculopathy secondary to the radiotherapy in the cervical or cerebral area that accelerates the process of thermogenesis of the blood vessels; (4) the traditional cardiovascular risk factors (hypertension, smoking, obesity, sedentarism which are partly risk factors for various neoplastic disorders [13,20-24].

The relationships of these mechanisms and how they progress to an ischemic stroke are not fully identified.

Recent research demonstrates that the pathogenesis of cancer associated thrombosis is multifactorial. It can be secondary to a cancer-induced procoagulant state, endothelial destruction due to surgical interventions. Moreover, chemotherapy's consequences may induce a hypercoagulability state following endothelial cell injury and the stimulation of inflammation [25].

Procoagulant factor production is a means by which the cancer induces a hypercoagulability state when neoplastic cells produce cancerous procoagulant which activates the factor $\mathrm{X}$ and fibrinogen $[26,27]$. 
Another hypothesis states that an increase in the tissue factor serum levels is one of the first initiators of the coagulation cascade. The micro-vesicles that contain and release tissue factor are the primary inductors that determine the hypercoagulation state. The studies demonstrated that neoplastic patients have significantly higher serum tissue factor levels compared to healthy controls. The serum tissue factor level in ovarian cancer significantly correlated with the risk of deep venous thrombosis $[28,29]$. Sinauridze et al. (2007) demonstrated that the micro-vesicles that arise from the platelets have a higher procoagulant activity, by 50 100-fold compared with activated platelets [30].

Cancer induces an inflammatory response with stimulation of pro-inflammatory cytokines, such as IL_6 and TNFa, cytokines that subsequently stimulate the production of procoagulant factors at the level of the endothelial cells (TF and von Willebrand factor). This actively contributes to a fibrin clot [31-34]. The inflammatory response determines the formation of neutrophil extracellular traps with a prothrombotic effect [35]. Cancerous patients also present with high levels of plasminogen activator inhibitor, which inhibits the plasminogen's activation and consequently inhibits the fibrinolysis [36].

In our study, the incidence of conventional cardiovascular risk factor (diabetes, smoking, AF) did not differ significantly between the two groups. These results are comparable with those reported by previous studies, in which no differences were found regarding stroke risk factors between non-cancerous and cancerous patients $[37,38]$. In contrast, in our group, the cholesterol, triglyceride and glycaemia levels were significantly lower for the SAC group. Other studies found that hypertension and smoking are the most frequent causes of stroke in cancer patients [3,39].

Chronic inflammation is dependent on the traditional cardiovascular risk factors, i.e.hypertension, dyslipidaemia, smoking, diabetes and obesity, adaptive immune mechanisms, i.e. production of pro-inflammatory cytokines, interferon $\gamma$ and TNF alpha, and innate immune mechanisms such as activation of toll receptor 4/6 pathways and IL-1 production [40]. Inflammation plays an important role in atherogenesis, and a decrease in inflammation is associated with a decrease in atherosclerotic processes.

The current study showed that the most frequent aetiologies of stroke in cancer patients, according to TOAST classification, were cryptogenic stroke, fol- lowed by large-artery atherosclerosis. In stroke patients without cancer, the most frequent aetiologies were large-artery atherosclerosis, followed by emboli of a cardiac origin.

Previous studies also demonstrated that stroke related cancer was of unknown cryptogenic aetiology [3, 41, 42]. Kim et al. (2010) demonstrated that in $40 \%$ of Korean stroke patients with cancer, the conventional stroke risk factors were absent and in this group of patients' multiple arterial territories were more frequently involved. This was considered suggestive of an embolic pattern of stroke due to cancer associated hypercoagulability [43].

The ESR is a nonspecific marker of infection and inflammation. Inflammation, a specific pathophysiological state in cancer, leads to coagulation and promotes progressive thrombogenesis. The ESR was considered a surrogate marker for inflammation in large and small vessel disease, related to atherosclerotic strokes [44]. In our study, the ESR was significantly increased in the SAC group, probably due to the presence of both neoplasia and stroke in these patients. The ESR level was higher in the SAC group. This occurrence might be secondary to neoplasia and stroke; the SAC patients presented with lower haemoglobin levels, which might partially explain an increased ESR for this group.

In the present study, stroke incidence was higher in patients with lung, colorectal, prostate, breast and bladder cancer. Schwarzbach et al. (2012) found that, in a German population, stroke was more frequent in patients with lung, colorectal, breast and prostate cancer. Ischemic stroke in neoplastic patients is more frequent in those with adenocarcinoma, especially pancreatic, lung and stomach $[6,45,46]$. One mechanism implicated in CAT in adenocarcinoma is the carcinoma mucins interaction with L- and P- selectins, thus determining a selected or dependent micro-angiopathy, which activates the coagulation cascade with an exaggerated thrombotic response through cancer procoagulants [47]. Thromboembolic cancer is a concept evolving from the perception that the $\mathrm{D}$-dimer levels were significantly higher in the group of patients with lung, pancreatic and gastric cancer $[6,46]$.

In the study group, the SAC patients had more severe neurological deficits and worse clinical outcomes than SWC patients. These results are consistent with those reported by Kneihsl et al. (2016), where the patients with active cancer had more severe strokes at admission, with a higher mortality rate during the hospi- 
talization [8]. Also, Cutting et al. (2016) demonstrated that almost half of stroke patients with cancer succumb in the first three months after a cerebrovascular event, and the survivors had a poor functional outcome [48].

It is known that hypercoagulability associated with cancer implies a poor prognosis for patients and an increased likelihood of mortality.

Lee et al. (2017) reported that in patients with CAT, the hypercoagulability state associated with cancer, assessed by D-dimers levels, was related to poor survival [17].

The correction of the hypercoagulability state, by the administration of parenteral anticoagulants, can play a protective role, with the report that there was an increased survival in anticoagulant-treated patients at one year after the diagnosis of CAT induced stroke [16].

In cancerous patients, the aetiology of stroke is frequently shadowed. The primary mechanism involved is most likely CAT, but numerous risk factors can subsequently influence the arterial thromboembolic risk in neoplastic patients. This risk of arterial thrombotic events associated with cancer has been underappreciated, and stroke in cancer patients carries a reserved clinical outcome, with more severe neurological deficits. The main concern is whether the risk of arterial thrombotic events associated with cancer are strongly linked with traditional cardiovascular risk factors or whether the cancer induced hypercoagulation plays the central role in the stroke pathogeny. This aspect is important for the significant therapeutic challenges that these patients are confronted with. Therefore, stroke induced by CAT should benefit from anticoagulant treatment.

\section{- CONCLUSIONS}

Stroke in cancer patients appears to be more frequently cryptogenic, probably due to cancer associated thrombosis. SAC patients had more severe neurological deficits and worse clinical outcomes than SWC patients.

This association between stroke and cancer should be kept reckoned with, especially in stroke of cryptogenic mechanism with embolic patterns, even in the presence of traditional cardiovascular risk factors.

\section{CONFLICT OF INTEREST}

None to declare.

\section{REFERENCES}

1. Rickles FR, Edwards RL. Activation of blood coagulation in cancer: Trousseau's syndrome revisited. Blood. 1983;62:1431.

2. Graus F, Rogers LR, Posner JB. Cerebrovascular complications in patients with cancer. Medicine. 1985; 64:16-35.

3. Cestari DM, Weine DM, Panageas KS, Segal AZ, DeAngelis LM. Stroke in patients with cancer: incidence and aetiology. Neurology. 2004;65: 2025-30.

4. Navi BB, Reiner AS, Kamel H, et al. association between incident cancer and subsequent stroke. Ann Neurol.2015;77:291-300.

5. Navi BB, ladecola C. Ischemic stroke in cancer patients: a review of an underappreciated pathology. Ann Neurol. 2018;83: 873-83.

6. Schwarzbach CJ, Schaefer A, Ebert A, et al. stroke and cancerthe importance of cancer-associated hypercoagulation as a possible stroke aetiology. Stroke.2012;43:3029-34.

7. Henley SJ, Singh SD, King J, Wilson RJ, O'Neil ME, Ryerson AB. Invasive cancer incidence and survival-United States, 2012. Morb Mortal Wkly Rep. 2015; 64:1353-58.

8. Kneihsl M, Enzinger C, Wunsch $G$, et al. Poor short-term outcome in patients with ischaemic stroke and active cancer. J Neurol. 2016; 263:150-56.

9. Taccone FS, Jeangette SM, Blecic SA. First-ever stroke as initial presentation of systemic cancer. J Stroke Cerebrovasc Dis. 2008;17: 169-74.

10. Bhattacharjee M, Vairale J, Gawali K, Dalal PM. Factors affecting burden on caregivers of stroke survivors: Populationbased study in Mumbai (India). Ann Indian Acad Neurol. 2012;15:113-119.

11. Stefan O, Vera N, Otto B, Heinz L,Wolfgang G. Stroke in cancer patients: a risk factor analysis. J Neurooncol.2009;94:221-26.

12. Balasa A, Chinezu R, Teleanu DM, Pascanu MI, Chinezu L, Borda A. Ectopic intracavernous corticotroph microadenoma: case report of an extremely rare pathology. Rom J Morphol Embryol. 2017;58:1447-1451.

13. Edwardson DW, Boudreau J, Mapletoft J, Lanner C, Kovala AT, Parissenti AM. Inflammatory cytokine production in tumour cells upon chemotherapy drug exposure or upon selection for drug resistance. PLoS One. 2017;12(9):e0183662.

14. Bamford J, Sandercock P, Dennis M, et al. A prospective study of acute cerebrovascular disease in the community: the Oxfordshire Community Stroke Project 1981-1986 1. Methodology, demography and incident cases of first-ever stroke. J Neurol Neurosurg Psychiatry 1988;51:1373-80.

15. Adams HP Jr, Bendixen BH, Kappelle LJ, et al. classification of subtype of acute ischemic stroke. Definitions for use in multicenter clinical trials. TOAST. Trial of Org 10172 in Acute Stroke Treatment. Stroke.1993;24:35-41.

16. Jang H-S, Choi J, Shin J, et al. The long-term effect of cancer on incident stroke: a nationwide population-based cohort study in Korea. Front Neurol.2019;10:52. 
Available online at: www.jccm.ro

17. Lee MJ, Chung JW, Ahn MJ, et al. Hypercoagulability and Mortality of Patients with Stroke and Active Cancer: The OASIS-CANCER Study. J Stroke. 2017;19(1):77-87.

18. Khorana AA, Francis CW, Culakova E, Fisher RI, Kuderer NM, Lyman GH. Thromboembolism in hospitalized neutropenic cancer patients. J Clin Oncol. 2006;24(3):484-90.

19. Selvik HA, Thomassen L, Bjerkreim AT, Naess H. Cancerassociated stroke: the Bergen NORSTROKE Study. Cerebrovasc Dis Extra.2015;5:107-113.

20. Dammacco F, Vacca A, Procaccio P, Ria R, Marech I, Racanelli $V$. Cancer-related coagulopathy: a review of literature and experience of a single centre of internal medicine. Clin Exp Med 2013;13:85-97.

21. Caine GJ, Stonelake PS, Lip GY, Kehoe ST. The hypercoagulable state of malignancy: pathogenesis and current debate. Neoplasia.2002;4:465-73.

22. Zuo PY, Chen XL, Liu YW, Xiao CL, Liu CY. Increased risk of cerebrovascular events in patients with cancer treated with bevacizumab: a meta-analysis. PloS One, 2014; 9: e102484.

23. Lysov Z, Dwivedi DJ, Gould TJ, Liaw PC. Procoagulant effects of lung cancer chemotherapy: impact on microparticles and cellfree DNA. Blood Coagul Fibrinolysis. 2017; 28:72-82.

24. Hung SK, Lee MS, Chiou WY, et al. High incidence of ischemic stroke occurrence in irradiated lung cancer patients: a population-based surgical cohort study. PloS One. 2014; 9:e94377.

25. Ay C, Pabinger I, Cohen AT. Cancer-associated venous thromboembolism: Burden, mechanisms, and management. Thromb Haemost. 2017;117(2):219-230.

26. Falanga A, Alessio MG, Donati MB, Barbui T. A new procoagulant in acute leukemia. Blood 1988; 71: 870-875.

27. Kazmierczak M, Lewandowski K, Wojtukiewicz MZ, et al. Cancer procoagulant in patients with adenocarcinomas. Blood Coagul Fibrinolysis 2005; 16: 543-547.

28. Ueno T, Toi M, Koike M, Nakamura S, Tominaga T. Tissue factor expression in breast cancer tissues: its correlation with prognosis and plasma concentration. Br J Cancer. 2000; 83: 164-170.

29. Uno K, Homma S, Satohet T, et al. Tissue factor expression as a possible determinant of thromboembolism in ovarian cancer. Br J Cancer. 2007; 96: 290-295.

30. Sinauridze El, Kireev DA, Popenko NY, et al. Platelet microparticle membranes have 50- to 100-fold higher specific procoagulant activity than activated platelets. Thromb Haemost. 2007; 97: 425-434.

31. Seruga B, Zhang H, Bernstein LJ, Tannock IF. Cytokines and their relationship to the symptoms and outcome of cancer. Nat Rev Cancer. 2008; 8: 887-899.

32. Kirchhofer D, Tschopp TB, Hadváry P, Baumgartner HR. Endothelial cells stimulated with tumor necrosis factoralpha express varying amounts of tissue factor resulting in
The Journal of Critical Care Medicine 2021;7(1) • 61

homogenous fibrin deposition in a native blood flow system. Effects of thrombin inhibitors. J Clin Invest. 1994; 93: 20732083.

33. Szotowski B, Antoniak S, Poller W, Schultheiss HP, Rauch U. Procoagulant soluble tissue factor is released from endothelial cells in response to inflammatory cytokines. Circ Res. 2005; 96: 1233-1239.

34. Bernardo A, Ball C, Nolasco L, Moake JF, Dong JF. Effects of inflammatory cytokines on the release and cleavage of the endothelial cell-derived ultralarge von Willebrand factor multimers underflow. Blood. 2004; 104: 100-106.

35. Fuchs TA, Brill A, Wagner DD. Neutrophil extracellular trap (NET) impact on deep vein thrombosis. Arterioscler Thromb Vasc Biol 2012; 32: 1777-1783.

36. Binder BR, Christ G, Gruber F, et al. Plasminogen activator inhibitor 1: physiological and pathophysiological roles. News Physiol Sci. 2002; 17: 56-61.

37. Grisold W, Oberndorfer S, Struhal W. Stroke and cancer: a review. Acta Neurol Scand.2009;119:1-16.

38. Zhang YY, Cordato D, Shen Q, Sheng AZ, Hung WT, Chan DK. Risk factor, pattern, etiology and outcome in ischemic stroke patients with cancer: a nested case-control study. Cerebrovasc Dis.2007;23:181-87.

39. Stefan O, Vera N, Otto B, Heinz L, Wolfgang G. Stroke in cancer patients: a risk factor analysis. J Neurooncol.2009;94:221-26.

40. Katsiari CG, Bogdanos DP, Sakkas LI. Inflammation and cardiovascular disease. World J Transl Med. 2019; 8(1): 1-8.

41. Kim K, Lee J-H. Risk factors and biomarkers of ischemic stroke in cancer patients. J Stroke.2014;16:91-6.

42. Nguyen T, DeAngelis LM. Stroke in cancer patients. Curr Neurol Neurosci Rep.2006;6:187-92.

43. Kim SG, Hong JM, Kim HY, et al. Ischemic stroke cancer in patients with and without conventional mechanism. Stroke. 2010;41:798-801.

44. Alvarez-Perez FJ, Castelo-Branco M, Alvarez-Sabin J. Usefulness of measurement of fibrinogen, D-dimer, D-dimer/fibrinogen ratio, $\mathrm{C}$ reactive protein and erythrocyte sedimentation rate to assess the pathophysiology and mechanism of ischemic stroke. J Neurol Neurosurg Psychiatry.2011;82:986-92.

45. Aarnio K, Joensuu $H$, Haapaniemi $E$, et al. Cancer in young adults with ischemic stroke. Stroke.2015;46:1601-6.

46. Chen PC, Muo CH, Lee YT, Yu YH, Sung FC. Lung cancer and incidence of stroke: a population-based cohort study. Stroke.2011;42:3034-39.

47. Bick RL. Cancer-associated thrombosis. N Engl J Med.2003;349:109-11.

48. Cutting S, Wettengel M, Conners JJ, Ouyang B, BusI K. Threemonths outcomes are poor in stroke patients with cancer despite acute stroke treatment. J Stroke Cerebrovasc Dis. 2017;26:809-15. 